\title{
Pneumologie 2005 - Future by Transition
}

\section{Liebe Leser,}

Der 46. Kongress der Deutschen Gesellschaft für Pneumologie 2005 in Berlin markiert eine weitere Periode erfolgreicher Jahre Arbeit unserer Fachgesellschaft.

Ein solcher Zeitpunkt bietet Gelegenheit, über die Vergangenheit, die Gegenwart und die Zukunft nachzudenken:

Die fast alles überdeckende Volkskrankheit Tuberkulose, deren Bekämpfung in der Nachkriegszeit eine klassische Erfolgsstory und Leitbild zur Bekämpfung aktueller Volkskrankheiten ist, hat in der Bundesrepublik heute einen eher überschaubaren gesundheitspolitischen Stellenwert. Heute, in einer Zeit der sozialen Emanzipation der breiten Massen, einer Zeit ohne verheerende Kriege, zumindest in Europa, und einer Zeit durchschnittlich wachsender Lebenserwartung hat sich die Lebenszeit der Menschen - von 35 Jahren im Bismarck'schen Preussen - verdoppelt und damit auch das Potenzial internistischer Erkrankungen. Das gilt auch und im Besonderen für Lungenkrankheiten. Während früher Infektionen wesentlich waren, steht heute die Suszeptivität der Lunge für Krankheiten infolge Zigarettenrauchen und Luftschadstoffe im Vordergrund. Folglich bilden die Chronischobstruktive Bronchitis (COPD) und das Bronchialkarzinom als raucherassoziierter Krebs profil- und strukturbestimmende Schwergewichte der klinischen Pneumologie. Daraus leiten sich wichtige Fragen für die pneumologische Forschung ab, die von der Präventiven Pneumologie bis hin zur Lungentransplantation reichen. Stellvertretend für viele, sei zentral eine Frage gestellt: „Wer wird vom Zigarettenrauchen krank werden?“ Diese Frage können wir heute immer noch nicht beantworten.

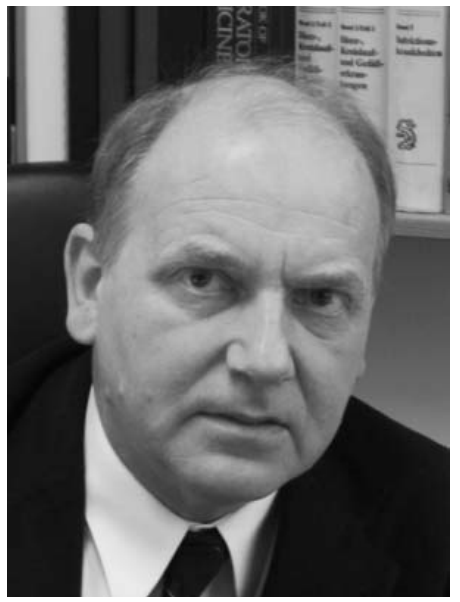

Prof. Dr. med. Christian Witt

Die Zukunft wird, wenn man den Annahmen der WHO folgt, einen erheblichen Zuwachs der Lungenkrankheiten bringen. In der Liste der zum Tode führenden Erkrankungen werden 2020 drei Lungenkrankheiten auf die vorderen Plätze rutschen: COPD, Bronchialkarzinom und Pneumonie. Dies stellt die Pneumologie vor große Aufgaben.

Zu deren Bewältigung bedarf es nicht nur einer Verbesserung der Strukturen, sondern auch eines verstärkten Wettbewerbes um die „besten Köpfe“. Hierzu muss eine hinreichende Präsenz der Pneumologie an den Medizinischen Fakultäten der Universitäten und lehrenden Pneumologischen Kliniken entwickelt werden. Im Wettbewerb um die Besten wird eine Verbesserung der wissenschaftlichen Strukturen der Pneumologie nützen. Das attrak- 
tive wissenschaftliche Feld wird engagierte junge Kollegen anziehen, es zu bestellen. Zusätzlich verbessert die wissenschaftliche Aktivität auch die Einbindung in den europäischen Kontext. Wissenschaftliche Aktivität und innovative Ergebnisse können die öffentliche Wahrnehmung der gesundheitspolitischen und -ökonomischen Bedeutung der Lungenkrankheiten und der gesamten Pneumologie fördern.

Der 46. Kongress der DGP 2005 in Berlin greift die Aufgaben auf und versucht in drei Schritten Antworten zu geben: Kompetenz erhöhen, Wissenschaft fördern, Gesundheits-, Wissenschaftsund Umweltpolitik aktiv mitgestalten.

Kompetenz erhöhen: Mit einer neu entwickelten Veranstaltungsreihe, dem Auditorium maximum, soll zu allen Teilgebieten der Pneumologie der aktuelle Stand in Wissenschaft und Ärztlicher Praxis vermittelt werden. Der Ort, der große historische Kuppelsaal des Kongresszentrums, spricht für die zentrale Position pneumologischer Fort- und Weiterbildung.

Wissenschaft fördern: Die pneumologische Forschung wird wesentlich differenzierter präsentiert. Zur Würdigung der wissenschaftlichen Arbeiten wird erstmals der Science-Preis an die besten Forscher vergeben. $\mathrm{Zu}$ dieser Preisverleihung werden die Erstautoren auf den traditionellen Campus der Charité zur Science-Party eingeladen. Platz gemacht hat hierfür das „in die Jahre gekommene“ sog. Referentenessen.

Gesundheits-, Wissenschafts- und Umweltpolitik aktiv mitgestalten: Der Kongress wird eröffnet durch die Bundesministerin für Gesundheit, dem Bürgermeister und Wirtschaftssenator von Berlin und Spitzen aus Medizin und forschender Arzneimittelin- dustrie. Diese öffentliche Wahrnehmung der Pneumologie, bzw. Ihrer Fachgesellschaft will ich für unser Fach nutzen. In einem Schwerpunkt wird auf den engen Zusammenhang zwischen Luftschadstoffen und Lungengesundheit eingegangen. Dieser Schwerpunkt soll auch in die breite Öffentlichkeit getragen werden. Dabei wird der Kongress aktiv durch das Bundesministerium für Umwelt und zahlreiche weitere Institutionen und Unternehmen unterstützt. Im neuen Format HOT TOPIC wird - angelehnt an die Struktur der Sendung „Sabine Christiansen“ - über aktuelle Probleme diskutiert. Ausgewählte Themen sind die gesundheitspolitische Bedeutung der EU-Osterweiterung, die Wissenschaftsförderung und die ökonomischen Perspektiven und ihre Konsequenzen. Nicht zuletzt soll im DGP-Damen-Lunch die Aktivität der weiblichen Mitglieder der DGP gewürdigt werden. Die Generalsekretärin der FDP Deutschlands, eine Sozialwissenschaftlerin der Humboldt-Universität zu Berlin und Lungenärztinnen unserer Gesellschaft werden zum Thema „In Zukunft mehr erfolgreiche Frauen (auch in der Lungenheilkunde)! - Wie macht man es besser?" diskutieren.

Wir treffen uns zu unserem Kongress in Berlins Mitte und das bedeutet mehr als eine geografische Marke. Die Botschaft heißt vielmehr, dass sich Klinik und Wissenschaft, auch jung und alt, Politik und Leben bis hin zu Ost und West treffen. Jede erfolgreiche Fachgesellschaft benötigt eine Vorstellung von sich selbst, eine Aufgabe und ein längerfristiges Ziel. Ich wünsche uns zu diesem 46. Kongress, dass wir gemeinsam erfolgreich an diesen Dingen arbeiten.

Prof. Dr. Christian Witt

Kongresspräsident 2005 